\title{
A escola promovendo conhecimento corporal como fortalecimento de políticas de saúde: uma revisão narrativa
}

\author{
The school promoting body knowledge as a strengthening of health policies: a narrative \\ review
}

\section{La escuela promotora del conocimiento corporal como fortalecimiento de las políticas de salud: una revisión narrativa}

\begin{abstract}
Lázaro Saluci Ramos ${ }^{1 *}$, Roseli Barreto da Silva², Jhonata da Silva Barreto², Mirian Belonia Mussi², Maria Vanderléia Saluci Ramos ${ }^{2}$, Andressilda Graça Santos Benevides ${ }^{2}$, Marciana dos Santos Silva Ventura $^{2}$, Claudiana do Nascimento ${ }^{2}$, Laura de Fátima Costalonga Ramos ${ }^{2}$, Marilda de Souza Pereira Bernardo ${ }^{2}$.
\end{abstract}

\section{RESUMO}

Objetivo: Trata-se de uma revisão de literatura tem como proposta agrupar a matéria que trata de políticas educacionais de conhecimento corporal com a finalidade de expor seus benefícios para a saúde dos alunos. Revisão bibliográfica: O corpo humano é a máquina mais complexa conhecida pela sociedade. É nele que se escondem as principais dúvidas da humanidade e os principais objetos de estudo das ciências globais. Desvendar o bom uso do corpo a fim de atender as necessidades humanas se tornou um objetivo vinculado a não objetificação dos seres humanos. A erotização do corpo se tornou um problema social para adolescentes que apresentam alterações naturais do corpo. A falta do conhecimento fez com que problemas como a gravidez na adolescência e a precoce contaminação por Doenças Sexualmente transmissíveis (DTSs) aumentassem consideravelmente. A escola, ambiente comum à grande maioria dos adolescentes brasileiros, abre-se como alternativa para modificar esta realidade. Considerações finais: $O$ estudo se inclina nas possibilidades através do ambiente escolar em ampliar a difusão de conhecimentos científicos e promover a anatomia como forma de combater de maneira preventiva problemas sociais da saúde adolescente.

Palavras-chave: Saúde do adolescente, Saúde na escola, Educação sexual.

\begin{abstract}
Objective: This is a review proposes to group the subject that deals with educational corporal education policies in order to expose its benefits to the students' health. Bibliographic review: The human body is the most complex machine known to society. It is there that the main doubts of humanity and the main objects of study of the global sciences hide. Unveiling the good use of the body in order to meet human needs has become an objective linked to the non objectification of human beings. The eroticization of the body has become a social problem for adolescents who have natural changes in the body. The lack of knowledge has caused problems such as teenage pregnancy and early contamination by Sexually Transmitted Diseases (STDs) to increase considerably. The school, an environment common to the vast majority of Brazilian adolescents, opens up as an alternative to modify this reality. Final considerations: The study leans on the possibilities through the school environment to expand the dissemination of scientific knowledge and to promote anatomy as a way of preventively combating social problems of adolescent health.
\end{abstract}

Key words: Adolescent health, School health, Sex education.

\footnotetext{
${ }^{1}$ Faculdade Multivix (MULTIVIX), Cachoeiro de Itapemirim - ES. *E-mail: lazaro321123@gmail.com

2 Faculdade Vale do Cricaré (FVC), São Mateus - ES.
} 


\section{RESUMEN}

Objetivo: Esta es una reseña que tiene como objetivo agrupar el tema que se ocupa de las políticas educativas sobre educación corporal para exponer sus beneficios a la salud de los estudiantes. Revisión bibliográfica: El cuerpo humano es la máquina más compleja conocida por la sociedad. Es allí donde se esconden las principales dudas de la humanidad y los principales objetos de estudio de las ciencias globales. Revelar el buen uso del cuerpo para satisfacer las necesidades humanas se ha convertido en un objetivo vinculado a la no objetivación de los seres humanos. La erotización del cuerpo se ha convertido en un problema social para los adolescentes que tienen cambios naturales en el cuerpo. La falta de conocimiento ha causado que problemas como el embarazo adolescente y la contaminación temprana por enfermedades de transmisión sexual (ETS) aumenten considerablemente. La escuela, un entorno común a la gran mayoría de los adolescentes brasileños, se abre como una alternativa para modificar esta realidad. Consideraciones finales: El estudio se apoya en las posibilidades a través del entorno escolar para expandir la difusión del conocimiento científico y promover la anatomía como una forma de prevenir los problemas sociales de salud de los adolescentes de manera preventiva.

Palabras clave: Salud del adolescente, Salud en las escuelas, Educación sexual.

\section{INTRODUÇÃO}

O indivíduo age no mundo através do seu corpo, mais especificamente através do movimento. É esse movimento físico que permite que as pessoas se comuniquem, trabalhem, aprendam, sintam o mundo e o aprecie. Sob a influência da aprendizagem e da experiência social e cultural, o sexo pode ser entendido através do desenvolvimento de disciplinas específicas, como biologia e ciências. Entretanto, a grande dificuldade de promover com qualidade o conhecimento corporal são os tabus que se formaram na sociedade ao longo dos milênios de evolução. Trata-se de aspecto cultural e social, que não pode ser alterado abruptamente, mas trabalhado dentro da compreensão e em consonância com a instrução social, evitando choques e repulsa (FURLANETTO MF, et al., 2018).

O sexo é um aspecto fundamental da vida humana, por isso não pode ser separado de outros aspectos da vida porque afeta pensamentos, sentimentos, comportamentos e interações. A construção desses conhecimentos é o resultado de várias socializações que uma pessoa experimenta na vida: família, escola, acesso a diferentes formas de comunicação, amizade e redes de vizinhança. Respeitada a individualidade humana e as questões materiais, há que se buscar uma forma de introduzir socialmente conhecimento sobre sexo e sexualidade. Os adolescentes seguem abandonados à margem e se tornando vulneráveis nessa questão. Os resultados acabam sendo terrivelmente irreversíveis, e em muitos casos, acabam se unindo a outros problemas como o desprezo social, e tendo efeitos mais pesados dependendo das particularidades de cada um (SILVA TRF, et al., 2019).

Afinal, sobre o que é gênero e sexualidade na escola? Qual o significado dos métodos para esses tópicos no universo escolar? Que afiliação ou características epistemológicas esse método usa? Por que devemos tratar de gênero e sexualidade nas instituições escolares? De certa forma, parte da sociedade brasileira reconhece o lugar do sexo nas escolas. Nas últimas duas décadas, após a epidemia do Vírus da Imunodeficiência Humana (HIV/AIDS) e o reconhecimento da gravidez de crianças em idade escolar, o sexo se tornou um local de palestras sobre o conceito de prevenção. No Brasil, o primeiro foco claro na educação sexual para crianças e jovens foi nas décadas de 1920 e 1930. Naquela época, a educação sexual havia se tornado uma preocupação para médicos, intelectuais, professores e professores da comunidade educacional brasileira na época (CÉSAR MRA, 2009).

Em 2010, um manuscrito didático foi distribuído pelo governo federal, que reabriu o debate sobre educação sexual no espaço escolar, recomendando que os professores fossem subsidiados no curso de instrução sobre sexo. O evento foi acompanhado de inúmeras críticas dos departamentos conservadores do poder legislativo e da sociedade civil, o que levou à coleta de materiais e atrasos na discussão do tema. A confusão foi 
tamanha, entretanto, não se pode considerar que a opinião pautada no senso comum possa interagir em pontos de discussão científica, de maneira satisfatória. Pouco foi tratado abertamente sobre as intenções reais do projeto, que giravam em torno do combate a sexualização infantil e da gravidez na adolescência (QUIRINO GS e ROCHA JBT, 2012).

A questão do sexo na escola, não somente se refere aos problemas tocantes a gravidez precoce ou Doenças Sexualmente Transmissíveis (DSTs), mas questões como o abuso sexual infantil e a exploração sexual infantil também podem ser trabalhados dentro da temática. Em localidades rurais, bairros distantes ou isolados, vivem uma grande parcela da população brasileira. Uma criança que não tem um contato social expandido, pode ter conhecimentos limitados no que se refere a sexo e sexualidade, o que dificultaria com que ela identificasse o abuso, que por vez pode ocorrer dentro da própria casa por membros da própria família, ou pessoas próximas. A intenção, que em nada se confunde com o ensino de atos ou da prática sexual, tem uma carga nobre, que é garantir a segurança e a consciência de crianças e adolescentes e a preservação da sua saúde física e mental (VERAS T, 2010).

Intenciona-se com este estudo, apontar a pesquisa já existente a fim de justificar a importância de o ambiente escolar promover o conhecimento corporal em alunos adolescentes. A desmistificação do sexo nas escolas, o combate a erotização do assunto e a derrubada de tabus construídos pela sociedade, também são objetivos da presente revisão. Conhecer e promover o conhecimento corporal pode ser tratado como política pública de saúde, já que está diretamente ligado ao combate da gravidez na adolescência, a prevenção de doenças sexualmente transmissíveis e a precaução contra danos causados a saúde mental e física do menor.

\section{REVISÃO BIBLIOGRÁFICA}

O sexo faz parte de toda a vida pessoal e, embora seja muitas vezes velado ou mal resolvido, não podemos deixar de mencionar ou tentar ignorá-lo. Ao resolver problemas sexuais com adolescentes, eles mostram uma variedade de conceitos, preocupações, expectativas e dúvidas ao longo da vida. Por isso, com base nessa narrativa é que a educação sexual não deve ser conduzida de maneira superficial e caótica, mas de maneira harmoniosa e saudável. O despertar sexual é acumulado e aprendido ao longo da vida porque faz parte do desenvolvimento humano, razão pela qual pode interferir em todo o processo de formação da personalidade. Os motivos para se incentivar o conhecimento do próprio corpo são bem construídos e compõem uma problemática singular (ALMEIDA ACCH e CENTA ML, 2009).

A discussão sobre como entendemos o corpo no processo de ensino sempre esteve cheia de um problema, ou seja, o entendimento de como somos educados. Para alcançar esse entendimento, devemos considerar que toda a educação é a educação do corpo e, além de adotar práticas contraditórias, ordens, uso de fórmulas e formas de medidas de contenção, também deve-se atender necessidades e desejos fisiológicos e adotar uma abordagem diferente. Como consideramos a posição do corpo na escola? Embora a característica da educação no Brasil seja a de que as crianças não estão prontas para a idade adulta, ensinando regras sociais no intuito de prepara-las, conhecer o corpo é recomendado como uma ferramenta necessária para o desenvolvimento cognitivo (ALMEIDA EB, et al., 2013).

A adolescência é um período importante na evolução dos seres humanos, durante esse período ocorrerão inúmeras mudanças no corpo, na psicologia, nas emoções e na sociedade, e ainda hoje é uma época em que os jovens experimentam desejo, dúvida, curiosidade e experimentaram tais mudanças. No processo de muitas mudanças, eles experimentaram o risco de descobrir que muitas vezes compartilham seu próprio prazer sexual e prazer sexual com seus parceiros, levando a uma gravidez inesperada. A vida sexual ativa entre adolescentes é uma realidade notória que requer conhecimento e orientação aos jovens para realizar sexo seguro, projetando-os para prevenir vários tipos de doenças sexualmente transmissíveis e gravidez não planejada (SILVA ACA, et al., 2013).

Para Silva R (2015), as famílias não são frequentemente usadas por jovens e adolescentes como fonte de informação sobre doenças sexuais e sexualmente transmissíveis. Estudos mostraram que é difícil para os pais discutir questões sexuais com filhos adolescentes, porque eles não têm espaço quando são jovens. 
Portanto, os pais atribuem esse papel à escola, que por sua vez acha difícil cumpri-lo, porque os professores geralmente sentem que não estão preparados. Por outro lado, consideramos a escola um exemplo importante de educação em saúde, devendo incluir tópicos como DST/AIDS, comportamento sexual e drogas. Examinando os Parâmetros Curriculares Nacionais (PCN) elaborados pelo Ministério da Educação (MEC), percebe-se que o tópico foi incluído no programa dentro de Temas Transversais.

O Brasil tem sido influenciado internacionalmente em termos de experiência e cuidado, principalmente na Europa. Incluindo políticas de planejamento familiar e educação sexual. Pode-se dizer que até o final do século XX o Brasil foi se tornado uma sociedade com um sistema normativo bem estabelecido para a proteção de adolescentes, e se mobiliza a participar de lutas e implementar políticas públicas para melhorar as condições de assistência à saúde, incluindo sexo e reprodução, e obter uso efetivo de métodos anti-gravidez. Ao longo do caminho histórico da sexualidade, sexo e educação sexual, fica claro que a hipótese em torno do sujeito é frequentemente uma moralidade restrita-conservadora, com pouca descrição de formas aceitas de comportamento (SFAIR SC, et al., 2015).

É compreensível, portanto, que o histórico do incentivo a conhecer o próprio corpo nas escolas possui um objetivo claro, não pornográfico, nobre e com intuito de prevenir sofrimento as futuras gerações. Muitos outros assuntos pertinentes podem ser apontados como justificativa a uma possível política nacional de educação sexual e conhecimento corporal. Não somente temas vinculados a DSTs e gravidez precoce, como também funcionamento biológico do corpo humano feminino, puberdade e outras questões vinculadas ao afloramento hormonal. Não existe outra forma mais eficaz de alcançar a resolução de problemas tão graves, senão pelo conhecimento e a instrução. Seria inocência acreditar que privar adolescentes do conhecimento sexual, no auge da explosão tecnológica, resolveria algum problema ou evitaria os danos possíveis (CECCARELLI PR e ANDRADE EL, 2018).

\section{A educação sexual derrubando tabus}

Acreditamos que a responsabilidade do sistema escolar é promover a educação abrangente de crianças e adolescentes, portanto, discutir o comportamento sexual para promover a educação sexual é uma iniciativa que deveria ter sido iniciada há muito tempo. O apelo sexual da mídia não é suficiente para permitir que os adolescentes adotem sexo seguro. A falta de educação sexual que vai além dos métodos tradicionais limita as perspectivas biológicas e médicas, que é uma das principais razões pelas quais os adolescentes não aderem ao sexo seguro. Qualquer trabalho com crianças ou adolescentes deve ser realizado continuamente, ou pelo menos dentro do prazo efetivo, para que, além de fornecer informações, as atitudes das pessoas em relação à coletividade e à individualidade também possam ser discutidas. Além disso, o trabalho deve ter características que começam com as dúvidas de crianças e jovens sobre os temas em que aparecem (MOIZÉS JS e BUENO SMV, 2010).

No relacionamento estabelecido entre alunos e professores, o poder do sexo é tão forte quanto os esforços das instituições educacionais para negar o sexo. A voz oficial da instituição escolar é em vão para garantir que qualquer evidência de conhecimento sobre comportamento sexual para professores e alunos não seja imediatamente suprimida. A situação das ideias sexuais entre alunos e professores é surpreendente e confusa, o que não é acidental, muitas vezes impresso nas manchetes, jornais, revistas e noticiários. A palavra tabu pode representar o nome exato do assunto: isso é algo misterioso, especialmente algo proibido. De qualquer forma, a regra padrão não deve ser violada, ou seja, quando algo acontece na sala de aula, professores e alunos devem se comprometer a se concentrar apenas em dados objetivos (ZUIN A, 2008).

A adolescência é uma etapa da vida humana, caracterizada por uma série de mudanças biológicas, psicológicas e sociais. É nesse estágio que a inserção sexual é usada como redescoberta das coisas inerentes ao ser humano e construída ao longo de sua trajetória pessoal, e seu exercício vai além da função reprodutiva e se estende ao processo de socialização dos adolescentes. Como todos sabemos, esse processo de amadurecimento trará certas vulnerabilidades, principalmente devido a mitos e tabus relacionados ao sexo, porque conceitos errados e mal-entendidos sobre sexo impedirão os adolescentes de viver completamente sem riscos (MARTINS CBG, et al., 2012). 
O surgimento do sexo nas ciências sociais aborda uma conexão entre cultura e subjetividade que surge de uma maneira particularmente complexa e incompreensível, o que parece resistir a qualquer forma de separação entre a sociedade e o espírito interior. Mesmo em alguns casos, essas áreas são consideradas como análises rigorosas e reconhecidas como artificiais. Quando pensamos mais especificamente sobre como a pedagogia sexual ou certo "conhecimento" eróticos se manifestam, eles são transferidos e eventualmente executados por indivíduos através de um processo complexo. É óbvio que é necessário estudar a apropriação indevida de pornografia e sexo com mais cuidado, evitando a confusão e o desgaste social pautado na baixa instrução (DUARTE LC e ROHDEN F, 2016).

O sexo é um tipo de estabelecimento e aprendizado. É uma parte indispensável do desenvolvimento da personalidade e pode interferir no processo de aprendizado da saúde mental e física pessoal. Portanto, entendemos que todas essas mudanças biológicas e psicológicas também levaram a mudanças na vida social. Esses elementos constituintes moldam a identidade dos adolescentes e é importante considerar, de alguma forma, uma visão geral dos processos sociais e culturais nos quais a identidade é construída. A identidade pode ser um fator central no gênero e no comportamento sexual, com o objetivo de identificá-lo como um processo em constante mudança e seu impacto nas experiências de vida sexual (BRÊTAS JRS, et al., 2011).

Portanto, toda pessoa tem sido associada a atitudes, valores, comportamentos e manifestações relacionadas ao sexo desde o nascimento, que são os elementos básicos do que chamamos de processo de educação sexual. Tem uma característica não intencional e existe desde o nascimento, inicialmente na família e depois em outros grupos sociais. Esta é a nossa maneira de valores construtivos e morais: consiste em religião, mídia, discurso literário, etc. A modificação desta realidade não parte apenas das estruturas sociais ou dos conceitos basilares da instituição familiar, mas antes dela, das instituições que conceituaram famílias e que promoveram o sexo algo pecaminoso e subversivo, no intuito de garantir controle sobre os atos humanos e construir um movimento de isolamento dos conhecimentos (MAIA ACB e RIBEIRO PRM, 2011).

\section{A política pública por detrás do conhecimento corporal}

A questão social por detrás do conhecimento corporal e desequilíbrio promovido pela sua ausência, faz com que assuntos de extrema importância sejam trazidos à baila, como a gravidez precoce, infecção por DSTs e combate ao abuso sexual infantil. Desde a década de 1970, as mães adolescentes são identificadas como questões de saúde pública. Complicações obstétricas e problemas psicológicos, sociais e econômicos que afetam mães e recém-nascidos apoiam essa afirmação. Resoluções baseadas em educação sexual, acesso a métodos contraceptivos e até abortos apoiam ações voltadas para a solução desse problema. A parte psicológica recai sobre a saúde mental, fragilizada pela quantidade de informações despejadas sobre uma adolescente, adultizando e forçando uma postura para a sociedade (PARIZ J, et al., 2012).

Durante a puberdade, a incidência de doenças sexualmente transmissíveis é maior: $25 \%$ dos jovens com menos de 25 anos são afetados por doenças sexualmente transmissíveis; $65 \%$ dos casos de Aids se manifestam entre 20 e 39 anos, refletidos na puberdade (período assintomático da doença $-10 / 15$ anos) infecção por HIV. Nos últimos anos, a incidência de DSTs na população em geral não mudou muito. Até testemunhamos a epidemia de gonococos e sífilis em todos os países desenvolvidos. Os principais motivos mencionados são biológicos, psicológicos e sociais. Em 2001, a OMS recomendou a substituição do termo DST por infecções sexualmente transmissíveis (IST), com o objetivo de enfatizar infecções assintomáticas. Durante a relação sexual, existem mais de 20 tipos de doenças infecciosas que se espalham facilmente (RODRIGUES MJ, 2010).

Todos os dias, novos casos de abuso e violência sexual são descobertos e relatados. Algumas notícias com maior influência social serão transmitidas repetidamente nas notícias, mídia impressa e eletrônica, e proporcionarão discussões nas mais diversas situações. Qualquer forma de comportamento sexual indesejável, tentativa, comentário ou insinuação, comercialização de seu sexo por coerção de outras pessoas ou outras maneiras de explorar seu sexo, independentemente de seu relacionamento com a vítima, em qualquer caso contra crianças e adolescentes são responsáveis por problemas de saúde pública no Brasil e 
no Mundo. Em todo o mundo, as notícias dos países africanos são as mais chocantes. Na República Democrática do Congo, a violência sexual é uma estratégia de guerra projetada para controlar a comunidade e insultar a família. As meninas são estupradas na frente de toda a comunidade, trazendo vergonha, medo e exclusão (PELISOLI C e PICCOLOTO LB, 2010).

Devido a essa série de problemas mais graves ocasionados pela ausência de conhecimento corporal e sexual, é que se vislumbra uma política pública sustentável, rentável e que possa enfrentar o problema gravíssimo em questão. Uma análise da implementação de políticas públicas no Brasil mostra a trajetória recente de desenvolvimento. Este é um campo consolidado com pouca acumulação de conhecimento. Portanto, como visa analisar a relação entre governo, funcionários do governo e cidadãos, entender o processo de implementação de políticas pode se traduzir em um fator importante na melhoria das ações do governo. É importante enfatizar que, na definição ampliada, o termo "governo" se aproxima dos países modernos, não apenas se referindo a grupos com poder político e definindo a orientação política de uma determinada sociedade, mas também a grupos com poder político institucionalmente (DALFIOR ET, et al., 2015).

Propor educação corporal nas escolas como política pública é a melhor maneira de propor a redução de problemas como a gravidez precoce, o abuso sexual infantil e a contaminação de jovens e adolescentes por doenças sexualmente transmissíveis. Apolítica pública é implementada em uma situação de caos e desordem social, onde os deveres do Estado não são cumpridos e recorrentes situações maléficas a sociedade acontecem devido a isso. É ímpar ter o conhecimento, e mais singular ainda é poder basear-se nele para combater um mal social (FURLANETTO MF, et al., 2018).

Em uma análise crítica é possível identificar os fatores que acumulam riscos aos jovens e adolescentes nas escolas, no que se refere a sexo e sexualidade. A adolescente que contrai uma gravidez indesejada, 0 adolescente que será pai precocemente, as doenças que ambos os grupos podem contrair além do abuso sexual que muitos são submetidos nos meios sociais que vivem. A instrução de jovens e adolescentes vai muito além de querer instrui-los, somente, para relações futuras, mas é uma ação direta de modificação social, que propõe uma mudança a longo prazo no comportamento e nos conceitos sociais, permitindo que esses problemas possam ser um dia extirpados da comunidade (VIANNA C, 2012).

A revisão das políticas públicas de educação a partir da perspectiva das relações de gênero e do contexto em que surgiram indica que o intenso processo de negociação determina a supressão e/ou implementação de reformas, planos, projetos e programas. Ações realizadas individual ou em conjunto pelo Estado e movimentos sociais que exigem novas políticas públicas, ocupam espaço na administração pública e reconhecem novas formas de desigualdade. Países e movimentos expressaram e/ou debateram ferozmente os interesses sociais que existiam nesse processo em sua respectiva diversidade. Nesse estágio em que deve haver conflitos e, às vezes, relações conflitantes, a formulação dessas políticas e a geração de conhecimento sobre elas levaram a discussões de complexidade (VIANNA C, 2012).

\section{CONSIDERAÇÕES FINAIS}

Considera-se, portanto, que a questão do conhecimento corporal é intimamente ligada a questão da educação social, ambos se completam e devem promover um ao outro formas de lidar com os problemas sociais graves gerados pela ausência do Estado nas questões discutidas. Uma política social de saúde está sempre voltada a tratar problemas que estão sendo banalizados socialmente, a gravidez na adolescência, o aumento dos casos de DSTs entre jovens e adolescentes além do abuso sexual infantil, podem ser combatidos dentro de sala de aula, com instrução, e formando mentes para que o futuro não tenha espaço para problemas tão graves. Não se trata de ensinar sexo ou promiscuidades, mas de garantir a segurança daqueles que devemos proteger.

\section{REFERÊNCIAS}

1. ALMEIDA ACCH, CENTA ML. A família e a educação sexual dos filhos: implicações para a enfermagem. Acta Paulista de Enfermagem, 2009; 22(1): 71-76. 
2. ALMEIDA EB, et al. O corpo como espaço de aprendizagem: reflexões a partir das vivências de um grupo de teatro formado por adultos na maturidade. Pro-Posições, 2013; 3(72): 201-217.

3. BRÊTAS JRS, et al. Aspectos da sexualidade na adolescência. Ciência \& Saúde Coletiva, 2011; 16(7): 3221 -3228.

4. CECCARELLI PR, ANDRADE EL. O sexual, a sexualidade e suas apresentações na atualidade. Revista Latinoamericana de Psicopatologia Fundamental, 2018; 21(2): 229-250.

5. CÉSAR MRA. Gênero, sexualidade e educação: notas para uma "Epistemologia". Educar em Revista, 2009; 35(1): 37-51.

6. DALFIOR ET, et al. Implementação de políticas públicas: metodologia de análise sob o enfoque da política institucional. Saúde e Debate, 2015; 39(esp): 283-297.

7. DUARTE LC, ROHDEN F. Entre o obsceno e o científico: pornografia, sexologia e a materialidade do sexo. Revista Estudos Feministas, 2016; 24(3): 715-737.

8. FURLANETTO MF, et al. Educação sexual em escolas brasileiras: revisão sistemática da literatura. Cadernos de Pesquisa, 2018; 48(168): 550-571.

9. MAIA ACB, RIBEIRO PRM. Educação sexual: princípios para a ação. Doxa, 2011; 15(1): 75-84.

10. MARTINS CBG, et al. Sexualidade na adolescência: mitos e tabus. Ciencia y enfermeira, 2012; 18(3): 25-37.

11. MOIZÉS JS, BUENO SMV. Compreensão sobre sexualidade e sexo nas escolas segundo professores do ensino fundamental. Revista da Escola de Enfermagem da USP, 2010; 44(1): 205-212.

12. $P A R I Z J$, et al. A atenção e o cuidado à gravidez na adolescência nos âmbitos familiar, político e na sociedade: uma revisão da literatura. Saúde e Sociedade, 2012; 21(3): 623-636.

13. PELISOLI C, PICCOLOTO LB. Prevenção do abuso sexual infantil: estratégias cognitivo-comportamentais na escola, na família e na comunidade. Revista Brasileira de Terapias Cognitivas, 2010; 6(1): 108-137.

14. QUIRINO GS, ROCHA JBT. Sexualidade e educação sexual na percepção docente. Educar em Revista, 2012; 43(1): 205-224.

15. RODRIGUES MJ. Doenças Sexualmente Transmissíveis (DST) na Adolescência. Nascer e Crescer, 2010; 19(3): 200 202.

16. SFAIR SC, et al. Educação sexual para adolescentes e jovens: mapeando proposições oficiais. Saúde Soc, 2015; 24(2): 620-632.

17. SILVA ACA, et al. Fatores de risco que contribuem para a ocorrência da gravidez na adolescência: revisão integrativa da literatura. Revista Cuidarte, 2013; 4(1): 531-539.

18. SILVA R. Quando a escola opera na conscientização dos jovens adolescentes no combate às DSTs. Educar em Revista, 2015; 57(2): 221-238.

19. SILVA TRF, et al. Representações dos estudantes de enfermagem sobre sexualidade: entre estereótipos e tabus. Trabalho, Educação e Saúde, 2019; 17(2): 1-15.

20. VERAS T. O Sistema Nacional de Combate ao Abuso e à Exploração Sexual Infantojuvenil e o Plano Nacional: um exemplo de política pública aplicada. Cadernos EBAPE.BR, 2010; 8(3): 404-421.

21. VIANNA C. Gênero, sexualidade e políticas públicas de educação: um diálogo com a produção acadêmica. ProPosições, 2012; 23(2): 127-143.

22. ZUIN A. Tabus sexuais entre professores e alunos. Pro-Posições, 2008; 19(2): 135-150. 